\title{
WEAK INTERACTION BREAKDOWN INDUCED BY SUPERGRAVITY
}

\author{
H.P. Nilles, M. Srednicki and D. Wyler \\ CERN - Geneva
}

\begin{abstract}
We show that spontaneously broken $\mathrm{N}=1$ supergravity can lead to an effective low-energy theory which is phenomenologically acceptable. We study a general low-energy theory and give restrictions which its parameters must satisfy in order to lead to a breakdown of weak interactions. The naturalness condition that the low-energy superpotential be scale invariant is imposed.
\end{abstract}

REF . TH . 3432-CERN

Ist October 1982 
Supergravity 1), with spontaneously broken local supersymmetry, can lead naturally to a small mass scale ${ }^{2), 3)}$. This scale is the gravitino mass, $\mathrm{m}_{3 / 2}$, and it is possible to have a large hierarchy between the Planck mass $M_{p}$ (and the grand unification mass $M_{x}$ ) and $m_{3} / 2$. In such a theory, physics at energies much less than $M_{p}$ can be described by an effective theory with softly broken global supersymmetry. This low-energy effective theory is specified by a superpotential and the type and magnitude of the soft breaking terms. We would like to explore the properties such a model must have in order to give a phenomenologically correct description of electroweak interactions. In particular, we want to have the breakdown of $S U(2) \times U(1)$ induced by the soft breaking terms.

We will impose a "naturalness" criterion on the low-energy superpotential. specifically, we will require it to be free of dimensionful parameters. Thus the only scale in the low-energy theory will be provided by the soft breaking terms, and will be of order $\mathrm{m}_{3 / 2}$. We believe that this is an important restriction which must be satisfied by any more complete theory in which parameters are not finely tuned.

We begin with a set of chiral supermultiplets and $\mathrm{SU}(3) \times \mathrm{SU}(2) \times \mathrm{U}(1)$ gauge fields coupled to $\mathrm{N}=1$ supergravity ${ }^{4), 5)}$. We will choose "minimal" kinetic terms ${ }^{5)}$. The superpotential is

$$
W=h\left(z_{i}\right)+g\left(y_{a}\right)
$$

Here the $z_{i}$ are scalar fields whose interactions will induce the spontaneous breakdown of supersymmetry, All the $z_{i}$ are chosen to be $\operatorname{SU}(3) \times \mathrm{SU}(2) \times \mathrm{U}(1)$ singlets. The $y_{a}$ include the quark, lepton, and Higgs scalars; the $y_{a}$ are the fields which will appear in the low energy theory ${ }^{F 1)}$. It is undoubtedly not necessary to maintain the strict separation between the $z_{i}$ and $y_{a}$ fields implied by Eq. (1). However, it greatly simplifies the analysis.

The scalar potential is ${ }^{5}$

$$
\begin{aligned}
V= & \exp \left[\left(\left|z_{i}\right|^{2}+|y a|^{2}\right) / M^{2}\right] x \\
& x\left[\left|h_{i}+z_{i}^{*} W / M^{2}\right|^{2}+\left|g_{a}+y_{a}^{*} W / M^{2}\right|^{2}-3|W|^{2} / M^{2}\right] \\
& +\frac{1}{2} D_{\alpha} D_{\alpha}
\end{aligned}
$$

where sums over $i, a$, and $\alpha$ are understood, and

$$
M=M_{P} / \sqrt{8 \pi}=2.4 \times 10^{18} \mathrm{GeV}
$$




$$
\begin{aligned}
& h_{i}=\partial h / \partial z_{i} \\
& g_{a}=\partial g / \partial y_{a} \\
& D_{\alpha}=e_{\alpha} \sum_{a} y_{a}^{*} T^{\alpha} y_{a}
\end{aligned}
$$

where $T^{\alpha}$ is a gauge group generator and $e_{\alpha}$ the associated coupling constant. We do not include a Fayet-Iliopoulos term 6 ).

We assume that $V$ is minimized when $z_{i}, h_{i}$, and $h$ have vacuum expectation values

$$
\begin{aligned}
\left\langle z_{i}\right\rangle & =b_{i} M \\
\left\langle h_{i}\right\rangle & =a_{i}^{*} m M \\
\langle h\rangle & =m M^{2} .
\end{aligned}
$$

Here $m$ is much less than $M$. The $y_{a}$, by assumption, have all negligeably small VEVs. There is no cosmological constant provided that. $V=0$ at the minimum;
this requires

$$
\sum_{i}\left|a_{i}+b_{i}\right|^{2}=3
$$

To construct the 1 ow-energy effective potential 7$), V_{L E}$, we replace $z_{i}$, $h_{i}$, and $h$ by their VEVs, and keep only those terms which do not vanish when $M \rightarrow \infty$. The result is $F 2$ )

$$
V=\left|\tilde{g}_{a}\right|^{2}+m_{3 / 2}^{2}\left|y_{a}\right|^{2}+m_{3 / 2}(A \tilde{g}+c . c .)+\frac{1}{2} D_{\alpha} D_{\alpha}
$$

where $\mathrm{m}_{3} / 2$ is the gravitino mass, given by

$$
m_{3 / 2}=\exp \left(\frac{1}{2}\left|b_{i}\right|^{2}\right) m
$$

$\tilde{\mathrm{g}}$ is the rescaled superpotential

$$
\tilde{g}=\exp \left(\frac{1}{2}\left|b_{i}\right|^{2}\right) g
$$

and the constant $A$ is given by

$$
A=b_{i}^{*}\left(a_{i}+b_{i}\right) \text {. }
$$


The fermionic part of the Lagrangian is that of a globally supersymetric theory

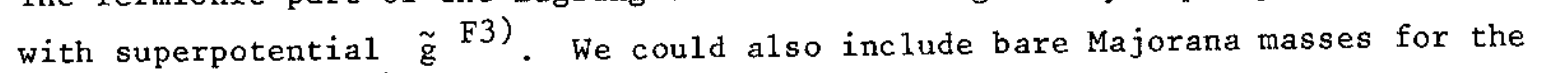
gauge fermions 2),3). They could arise from one loop graphs involving the gravitino 8), or from non-minimal kinetic terms for the gauge fields 5),9). Any such gauge fermion mass should be of order $m_{3} / 2$.

Now let us analyze $V_{L E}$. If $|A|<3, V_{L E}$ is positive definite and has a minimum at $y_{a}=0$. To see this, note that

$$
\left|\tilde{g}_{a} \pm m_{3 / 2} y_{a}^{*}\right|^{2}=\left|\tilde{g}_{a}\right|^{2}+m_{3 / 2}^{2}\left|y_{a}\right|^{2} \pm 3 m_{3 / 2}\left(\tilde{g}+\tilde{g}^{*}\right)
$$

The left-hand side is positive definite, while the right-hand side is less than $V_{L E}$ if $|A|<3$. So only theories with $|A|>3$ can result in spontaneous breakdown of $\mathrm{SU}(2) \times \mathrm{U}(1)$ at the tree level.

Let us assume that $A$ is real and greater than 3 (1ater we will see that this is possible), and choose

$$
\tilde{g}=\lambda Y H \bar{H}+\frac{1}{3} \sigma Y^{3}
$$

where $H$ and $\bar{H}$ are SU(2) doublets of opposite hypercharge, and $Y$ is an $\mathrm{SU}(2) \times \mathrm{U}(1)$ singlet. This is the simplest superpotential which can result in spontaneous breakdown of $\mathrm{SU}(2) \times \mathrm{U}(1)$. Since $A>3, \mathrm{~V}_{\mathrm{LE}}$ is no longer positive definite and the absolute minimum will be obtained for non-vanishing VEVs of the $\mathrm{Y}$ and/or $\mathrm{H}, \overline{\mathrm{H}}$ scalars. For example, in the range of parameters

$$
1<\frac{\sigma}{\lambda}<\frac{1}{16}\left(A+\sqrt{A^{2}-8}\right)^{2}
$$

there is an absolute minimum (with $V_{L E}<0$ ) where $Y$ and the neutral components of the $\mathrm{H}$ and $\overline{\mathrm{H}}$ doublets have non-vanishing VEVs. $\langle\mathrm{H}\rangle$ and $\langle\overline{\mathrm{H}}\rangle^{*}$ will be equal to minimize the $\mathrm{D}^{2}$ term in the potential.

One has, of course, to include the scalar quark and lepton fields in the lowenergy superpotential, and one might worry that at the global minimum of the full theory these fields could have non-vanishing VEVs as well. This, however, does not happen if the gravitino mass is larger than the masses of quarks and leptons. The reason is the presence of the term $\mathrm{m}_{3 / 2}^{2}\left|\mathrm{y}_{\mathrm{a}}\right|^{2}$ in the potential which forbids VEVs for the partners of quarks and leptons as long as the product of $A$ and each Yukawa coupling is not too large. The potential $V_{L E}$ thus has all the desired properties if 


\section{$3<|A|<m_{3 / 2} / m_{t}$}

where $m_{t}$ is the mass of the heaviest quark or lepton. The upper bound on $|A|$ is sufficient to ensure that quark and lepton scalars have vanishing VEVs, but is probably too strict.

Thus in this model it is possible to have a global minimum of the low-energy potential with the correct pattern of gauge symmetry breakdown, provided $\mathrm{m}_{3} / 2$ is of the order of $\mathrm{M}_{W}$. All the scalar partners of quarks and leptons have masses of order $\mathrm{m}_{3 / 2}$. The form of the scalar mass matrix eliminates flavour-changing neutral currents, as desired.

Finally, we have studied a superpotential for a single $z$ field of the form $h(z)=m M(z+B M)$ to see if $|A|>3$ is, indeed, possible. If we insist on zero cosmological constant, then $|A|=3-\sqrt{3} F 4)$. However, we find that there is a local minimum of the potential with $|A|>3$, provided $27 / 2<\beta^{2}<16^{\text {F5 })}$. Unfortunately, this minimum is not global and has a non-zero cosmological constant, indicating the need for more complicated $h\left(z_{i}\right)$.

To summarize, we find that spontaneously broken supergravity results in an effective low-energy theory which has softly broken global supersymmetry. The scale of the soft breaking is the gravitino mass, $\mathrm{m}_{3} / 2$. We impose the natura1ness condition that the only scale in the low-energy theory is provided by the soft breaking terms and find that phenomenologically consistent models of the electroweak interactions are possible, provided certain restrictions are imposed on the underlying theory (i.e., it must yield $|\mathrm{A}|>3$ ). Radiative corrections will not disturb this picture since all couplings are weak and all scalars have laxge $\left(\sim M_{W}\right)$ bare masses.

Other authors have considered grand unified theories coupled to $\mathrm{N}=1$ supergravity. In Ref. 9), the low-energy theory is not analyzed. In Refs. 7) and 10) explicit mass parameters are introduced into the low-energy superpotential.

\section{ACKNOWLEDGEMENTS}

We are grateful to F. Bopp Jr. for help with the numerical calculations. We enjoyed discussions on inequalities with H. Grosse. 


\section{FOOTNOTES}

F1) Some of the $z_{i}$ may also be 1ight, but their interactions with the $y_{a}$ will be suppressed by inverse powers of $\mathrm{M}_{\mathrm{p}}$. We will ignore any such light $z_{i}$, as they are phenomenologically irrelevant.

F2) To obtain this result we used $\sum_{a} g_{a} y_{a}=3 g$.

F3) These results have been obtained in the special case of one $z$ field and $h(z)=m M[z+(2-\sqrt{3}) M]$ by Barbieri, Ferrara, and Savoy 7$)$. In this case, $A=3-\sqrt{3}$.

F4) Nath, Arnowitt and Chamseddine ${ }^{10)}$ claim that $A=3+\sqrt{3}$ is possible for this choice of $h(z)$. However, the extremum of the potential that yields $A=3+\sqrt{3}$ (with vanishing cosmological constant) is actually a saddle point, not a minimum.

F5) For $B^{2}$ in this range, $z / M$ takes on values between -2.2 and -2 . These results are got by solving the extremum equation of the potential, $z^{3}+z^{2} \beta-2 \beta=0, \quad z \varepsilon R$. 


\section{REFERENCES}

1) For a review see : P. van Nieuwenhuizen, Physics Reports 68 (1981) 189.

2) H.P. Nilles, Physics Letters 115B (1982) 193, and Proceedings of the Johns Hopkins Workshop on Current Problems in Particle Theory 6, eds. G. Domokos et al., Johns Hopkins Univ. Press, Baltimore (1982).

3) H.P. Nilles, CERN preprint.TH. 3398 (1982).

4) E. Cremer, B. Julia, J. Scherk, S. Ferrara, L. Girardello and P. van Nieuwenhuizen, Nuclear Physics B147 (1979) 105.

5) E. Cremex, S. Ferrara, L. Girarde110 and A. Van Proeyen, CERN preprints TH. 3312 and 3348 (1982).

6) P. Fayet and J. Iliopoulos, Phys. Letters 31B (1974) 461.

7) R. Barbieri, S. Ferrara and C. Savoy, CERN preprint TH. 3365 (1982).

8) R. Barbieri, S. Ferrara, D. Nanopoulos and K. Stelle, Phys. Letters 113B (1982) 219.

9) L. Ibañez, CERN preprint TH. 3374 (1982).

10) A.H. Chamseddine, R. Arnowitt and P. Nath, Northeastern University preprints 2559 and 2565 (1982). 